Wolfgang Marquardt

\title{
Urheberrecht und Open Access: Angemessene Rahmenbedingungen für die Wissenschaft ${ }^{1}$
}

\begin{abstract}
Zusammenfassung: Wissenschaftliche Arbeitsumgebungen werden zunehmend digital. Dafür benötigen wir geeignete rechtliche und infrastrukturelle Rahmenbedingungen. Die Allianz der Wissenschaftsorganisationen setzt sich in einer Schwerpunktinitiative umfassend für einen möglichst entgelt- und barrierefreien Zugang zu digitalen Publikationen und Forschungsprimärdaten ein. Eine zentrale Forderung der Allianz ist das unabdingbare Zweitverwertungsrecht für wissenschaftliche Autoren. Außerdem bedarf es erheblicher Investitionen von Bund und Ländern in die systematische Weiterentwicklung der Informationsinfrastrukturen.
\end{abstract}

Schlüsselwörter: Open Access; Urheberrecht; Informationsinfrastruktur; Nutzungsrechte

\section{Copyright and Open Access: Appropriate Basic Conditions for Sciences}

Abstract: Scientific work environments become increasingly digitally. For this fact we need suitable legal and infrastructural basic conditions. The Alliance of Scientific Organisations stands up by a main focus initiative for an access by fee free and free of barriers to digital publications and primary research data. A central claim of the Alliance is the inalienable secondary exploitation right for scientific authors. Besides this considerable investments by federal and state governments in the systematic further development of the information infrastructures are necessary.

Keywords: Open access; copyright; information infrastructure; usage rights

Prof. Dr.-Ing. Wolfgang Marquardt:

Wolfgang.Marquardt@avt.rwth-aachen.de

1 Vortrag anlässlich der Veranstaltung „Urheberrecht für die Wissensgesellschaft - Herausforderungen in der digitalen Welt“ des Deutschen Bibliotheksverbandes e.V. (dbv) am 25. Oktober 2012 in Berlin.

\section{Einführung}

Das Urheberrecht ist ein Thema, das zunächst einmal als recht trockenes Spezialistenthema daherkommt, mit dem sich in früheren Zeiten vornehmlich Bibliothekare, Archivare und Urheberrechtsjuristen stellvertretend für uns Wissenschaftler als Urheber und Nutzer wissenschaftlicher Literatur beschäftigt haben.

In den letzten Jahren hat sich das Urheberrecht jedoch immer weiter ins Gesichtsfeld der Wissenschaft, der Wissenschaftsorganisationen und auch der Öffentlichkeit gedrängt. Wissenschaftliche Arbeitsumgebungen werden in rasantem Tempo digital. Dafür benötigen wir geeignete rechtliche und infrastrukturelle Rahmenbedingungen, die bald gesetzt werden müssen, um im internationalen Wettbewerb bestehen zu können. Aufgrund der rasanten Entwicklungen im Bereich E-Science erfolgen alle systemischen Anpassungen zwangsläufig zeitverzögert. Die Baustelle mit dem größten Trägheitsmoment ist dabei eine adäquate Novellierung des Urheberrechtsgesetzes. Obwohl es mittlerweile bereits zweimal mit Blick auf die digitalen Medien novelliert wurde, befindet es sich in Bezug auf die Belange der Wissenschaft noch im Postkutschenzeitalter. Außerdem ist es schwer verständlich und zugänglich. Dies hat sogar Ministerin Leutheusser-Schnarrenberger in einem Beitrag in der Frankfurter Allgemeinen Zeitung im Sommer dieses Jahres eingeräumt. ${ }^{2}$ Doch es geht weniger um die von der Ministerin benannten „Akzeptanzprobleme“ als Folge der „Verkomplizierungen“ des Urheberrechtsgesetzes, als vielmehr um ungewollte Verletzungen des geltenden Rechts oder um den präventiven Verzicht seiner Anwendung aus Angst vor einer möglichen Rechtsverletzung. Dies war z. B. im Falle der elektronischen Semesterapparate zu beobachten, die nur sehr zögerlich eingerichtet wurden, obwohl die technischen Möglichkeiten schon frühzeitig zur Verfügung standen.

2 Sabine Leutheusser-Schnarrenberger: Kein Grund zum Kulturpessimismus, Frankfurter Allgemeine Zeitung vom 31.05. 2012: „Mit immer weiteren Gesetzen kann der Schutz des geistigen Eigentums nicht verbessert werden, sondern es droht auch die Gefahr der Verschlechterung. So haben die letzten gesetzlichen Änderungen zwischen 1998 und 2009 zu erheblichen Verkomplizierungen am Text des Urheberrechtsgesetzes und deutlichen Akzeptanzproblemen geführt.“ 
Ein der Wissenschaft gewidmeter Dritter Korb des Urheberrechts wurde vor Jahren angekündigt ${ }^{3}$, aber bisher nicht realisiert. Entsprechend hoch ist mittlerweile der Druck aus der Wissenschaft. Die Allianz der Wissenschaftsorganisationen ${ }^{4}$, das Aktionsbündnis Urheberrecht für Bildung und Wissenschaft ${ }^{5}$, der $\mathrm{dbv}^{6}$ und auch die Kultusministerkonferenz ${ }^{7}$ formulieren daher seit Jahren neben anderen Akteuren immer wieder ihre Anforderungen an ein wissenschaftsfreundliches Urheberrecht. Eine Enquete-Kommission ${ }^{8}$ des Deutschen Bundestags veröffentlicht Handlungsempfehlungen im Sinne der Positionen der Wissenschaft, alle Bundestagsfraktionen und Parteien mit Ausnahme der FDP melden sich mit Vorschlägen zu Wort, die mehr oder weniger nah an den Vorstellungen der Wissenschaft zur Novellierung des Urheberrechtsgesetzes orientiert sind und eine umfassende Open-Access-Strategie einfordern. Das Bundesministerium der Justiz (BMJ) und das Bundesministerium für Bildung und Forschung (BMBF) organisieren Anhörungen und Gesprächsrunden. Aber was geschieht? Der Berg kreißt seit Jahren und gebiert ... nein, keine Maus, sondern leider nur ein Leistungsschutzschutzrecht für Presseverlage.

Das BMJ scheint nicht in der Lage, gegen die mächtige Lobby der Wissenschaftsverlage einen mutigen Schritt für die Wissenschaft zu gehen. Es nimmt in Kauf, dass die öffentliche Hand überwiegend die Forschung finanziert, anschließend für den Zugang zu den daraus resultierenden Forschungsergebnisse teils völlig inakzeptable Preise an die Wissenschaftsverlage bezahlt, um dann am Ende

3 Der Bundesrat hatte bereits im Jahre 2006 ein „bildungs- und wissenschaftsfreundlicheres Urheberrecht“ bzw. ein dezidiertes Wissenschafts-Urheberrecht gefordert. Diesem Wunsch hatte sich der Deutsche Bundestag angeschlossen und in seiner letzten Beschlussempfehlung zum Zweiten Korb das Bundesjustizministerium z. B. um die „Prüfung eines Zweitverwertungsrechts für Urheber von wissenschaftlichen Beiträgen, die überwiegend im Rahmen einer mit öffentlichen Mitteln finanzierten Lehr- und Forschungstätigkeit entstanden sind ( $\$ 38$ UrhG)" gebeten.

4 Allianz der Wissenschaftsorganisationen: Neuregelung des Urheberrechts: Desiderate für einen Dritten Korb, 9. Juli 2010.

5 Aktionsbündnis Urheberecht für Bildung und Wissenschaft: Vorschlag einer allgemeinen Wissenschaftsschranke, vgl. Pressemitteilung des Aktionsbündnisses 06/10 vom 6. Juli 2010 (http://www. urheberrechtsbuendnis.de/pressemitteilung0610.html.de).

6 dbv-Stellungnahme zum Urheberrecht "Nutzerinteressen stärken, Urheberrechte wahren" vom 20. 04. 2012.

7 Kultusministerkonferenz: Positionspapier der Kultusministerkonferenz zur Prüfung weiteren gesetzlichen Handlungsbedarfs im Bereich des Urheberrecht, Bonn, 08. 06. 2009.

8 Deutscher Bundestag, Enquete-Kommission Internet und digitale Gesellschaft, Projektgruppe Bildung und Forschung: Handlungsempfehlungen, Ausschussdrucksache 17(24)052 mit Stand vom 25. 06. 2012 des Tages doch nicht mehr in der Lage zu sein, die Informationsversorgung flächendeckend $\mathrm{zu}$ garantieren. Es schafft keinen fairen Ausgleich zwischen den Interessen der Rechteverwerter einerseits und der Urheber und Nutzer wissenschaftlicher Informationen andererseits. ${ }^{9}$ Selbst eine rechtzeitige Initiative zur Entfristung oder zumindest zur fortgesetzten Befristung des Paragraphen 52 a UrhG über das Ende des Jahres 2012 hinaus ist das BMJ trotz einer gegenteiligen schriftlichen Versicherung der Ministerin schuldig geblieben. Sie wurde im Oktober 2012 nun auch noch vom Bundesrat dringlich angemahnt. ${ }^{10}$

Bereits jetzt gibt es aufgrund der Unzulänglichkeiten des geltenden Urheberrechtsgesetzes eine erhebliche Frustration in der Wissenschaft, die sich bisweilen sogar im Aufruf zum zivilen Ungehorsam Bahn bricht. Ziel dieser Aufforderung ist es, die Gerichte bis hin zur obersten Instanz mit Musterprozessen zu beschäftigen, um den Gesetzgeber zu einer Novellierung des Urheberrechtsgesetzes zu zwingen. Leider geben die vorliegenden Urteile in den bisher unterinstanzlich geführten Verfahren hier keinen Anlass zu besonderem Optimismus für die Belange der Wissenschaft.

Das ist - sehr kurz gefasst - der betrübliche Stand der Dinge. Dennoch sollten wir alle nicht müde werden und weiterhin für ein wissenschaftsfreundliches Urheberrecht in Deutschland und auch in Europa kämpfen. In Brüssel nimmt - wie Sie alle wissen - gerade die zuständige Kommissarin Nelly Kroes einen hoffnungsvoll stimmenden An-

9 Vgl. hierzu auch meinen Artikel „Fairness und Ausgewogenheit im Urheberrecht“ in der Frankfurter Allgemeinen Zeitung vom 08.08. 2012.

10 Der Deutsche Bundestag hat schließlich am 29. November 2012 den von den Fraktionen der CDU/CSU und FDP eingebrachten Entwurf eines Gesetzes zur Änderung des Urheberrechtsgesetzes angenommen und einer weiteren Verlängerung der Befristung des $\S 52 \mathrm{a}$ UrhG bis zum 31.12.2014 zugestimmt. Der Bundesrat hat am 14.12. 2012 der Verlängerung der Befristung des § 52a UrhG ebenfalls zugestimmt. Dabei nimmt er mit Bedauern zur Kenntnis, dass der Deutsche Bundestag der Forderung der Länder nach einer vollständigen Entfristung nicht gefolgt ist: „Die erneute Verlängerung der Befristung um zwei Jahre ist der weniger geeignete Weg, diese Sicherheit [im digitalen Umgang mit urheberrechtlich geschützten Materialien] herzustellen, zumal keine Perspektive erkennbar ist, durch welche Norm der § 52a UrhG nach Auslaufen ersetzt werden soll. Die nun vierte Befristung von § 52a UrhG ist einer Rechtssicherheit im Umgang mit urheberrechtlich geschützten Materialien im gesamten Bildungsbereich nicht förderlich. [...] Der Bundesrat geht davon aus, dass die Bundesregierung unverzüglich und in enger Abstimmung mit den Ländern die Arbeiten an einer breiter und allgemeiner gefassten Bildungs- und Wissenschaftsschranke aufnimmt, wie sie einvernehmlich von Kultusministerkonferenz und Wissenschaftsallianz gefordert wird.“ Bundesrat, Drucksache 737/12 (Beschluss) vom 14.12. 2012 zur 904. Sitzung des Bundesrates. 
lauf zu einer überarbeiteten EU-Richtlinie. Doch es gibt auch Anzeichen dafür, dass andere am Thema Copyright beteiligte Kommissare, Barnier und Vassiliou, die Uhren eher in Richtung auf eine weitestgehende Lizenzierung zwischen Verlagen und Nutzern stellen könnten, statt in Richtung auf weite und klare gesetzliche Schrankenregelungen. Auch hier ist nicht transparent, ob und wie sich deutsche Regierungsvertreter für wissenschaftsfreundliche Regelungen einsetzen.

\section{Maßnahmen der Allianz der Wissenschaftsorganisationen}

Im Folgenden möchte ich Ihnen berichten, was die Allianz der Wissenschaftsorganisationen in ihrer Schwerpunktinitiative „Digitale Information“ unternimmt, um Open Access im Sinne der von uns unterzeichneten Berliner Erklärung zu befördern und das Urheberrecht zu reformieren. Die 2008 aufgesetzte Schwerpunktinitiative ${ }^{11}$ hat sich zum Ziel gesetzt, Wissenschaftlerinnen und Wissenschaftlern einen möglichst entgelt- und barrierefreien Zugang zu digitalen Publikationen und Forschungsprimärdaten $\mathrm{zu}$ ermöglichen. Außerdem soll eine integrierte digitale Forschungsumgebung geschaffen werden, in der jeder Forschende überall in Deutschland auf alle relevanten wissenschaftlichen Publikationen und Forschungsprimärdaten zugreifen kann. Die Allianz-Organisationen verpflichten sich, ihre Kompetenzen und Ressourcen in sechs Arbeitsbereichen zu bündeln sowie aktuelle und zukünftige Aktivitäten stärker zu koordinieren. Diese sind

- nationale Lizenzierungen

- Open Access

- nationale Hosting-Strategien

- Forschungsprimärdaten

- virtuelle Forschungsumgebungen

- rechtliche Rahmenbedingungen

Ich möchte mich hier beispielhaft auf drei der sechs Felder, nämlich Open Access, Forschungsprimärdaten und Rechtliche Rahmenbedingungen, konzentrieren.

\subsection{Open Access}

Ziel von Open Access ist es, Voraussetzungen dafür zu schaffen, das wissenschaftliche Wissen, das in digitaler Form vor-

11 Vgl. www.allianzinitiative.de. liegt, ohne finanzielle, technische oder rechtliche Barrieren zugänglich und nachnutzbar zu machen.

Die zu Open Access erarbeitete einheitliche Positionierung der Allianz der Wissenschaftsorganisationen ist sowohl in der Politik wie auch bei internationalen Partnern interessiert wahrgenommen worden. Mit der Einführung einer Open-Access-Komponente in Allianz-Lizenzen wird beispielsweise ein wesentlicher Baustein für den Paradigmen-Wechsel hin zu Open Access in der Fläche angestrebt. Mit Handreichungen und Broschüren werden außerdem wichtige Impulse zum Umgang mit Open Access an Hochschulen und Forschungseinrichtungen gegeben. Künftige Empfehlungen und Handreichungen werden stärker die konkret-operative Umsetzung von Open Access durch Hochschulen und Forschungseinrichtungen adressieren.

Neben dem Goldenen Weg zu Open Access verfolgt die Allianz weiterhin den Grünen Weg. Auf dem Weg zu Gold sind noch umfangreiche Vorarbeiten zu erledigen. Dazu gehören die Klärung des Verhältnisses von Publikationsgebühren zu Subskriptionspreisen und die Erarbeitung von Kriterien für die Übernahme von Publikationsgebühren. Erforderlich sind aber auch Mindeststandards zur Gestaltung von Nutzungsrechten an Publikationen, damit sichergestellt ist, dass die Modalitäten einer (Nach-) Nutzung digitaler Inhalte wieder in die Hand der Wissenschaft und der sie mit Informationen versorgenden Bibliotheken kommen. Und - last but not least - bedarf es einer nationalen Hosting-Strategie, um die dauerhafte Verfügbarkeit digitaler Publikationen - auch über die Existenz eines Verlagshauses hinweg - zu gewährleisten.

\subsection{Forschungsprimärdaten}

Ein weiteres Ziel ist es, wissenschaftsgeleitet die notwendigen Prozesse einzuleiten, die es erlauben, fachspezifisch nutzbare Infrastrukturen zum Management von Forschungsprimärdaten zu entwickeln und zu implementieren.

Mit dem Policy-Papier der Allianz zum Umgang mit Forschungsdaten liegt eine Richtschnur für die weiteren Maßnahmen in den nächsten Jahren vor. Künftig wird es im Wesentlichen um das Forschungsdatenmanagement, ${ }^{12}$ um die Nachnutzung und Verfügbarkeit von Forschungsdaten, um die Abschätzung der Kosten zum Aufbau, zur

12 Dies umfasst die Erstellung einer Checkliste zum Datenmanagement, die Erarbeitung von Empfehlungen zu Zitierweisen von Forschungsdaten und die Formulierung eines Vorschlags zu minimalen Anforderungen an Metadaten zur Verbesserung der Dateninteroperabilität und des Austauschs von Metadaten. 
Entwicklung und zum Betrieb einiger ausgewählter Forschungsdatenzentren in unterschiedlichen Fachdisziplinen wie auch um die rechtlichen Rahmenbedingungen für die Bereitstellung von Forschungsdaten gehen. Neben der Klärung grundsätzlicher Fragen - wie z. B. der Urheberrechte an Forschungsdaten - sollen hier vor allem Mechanismen und Regelungen entwickelt werden, die den freien Zugang zu Forschungsdaten und ihre möglichst uneingeschränkte Nutzung für wissenschaftliche Zwecke gewährleisten. Klar ist, dass die Rechte an Forschungsdaten nicht vollständig an kommerzielle Verlage vergeben werden dürfen.

\subsection{Rechtliche Rahmenbedingungen}

Die Ziele im Arbeitsbereich Rechtliche Rahmenbedingungen sind ein wissenschaftsfreundliches Urheberrecht und die Verminderung des Mehrwertsteuer-Satzes für digitale Publikationen.

Bislang stand die Novellierung des Urheberrechts im Vordergrund der Aktivitäten. Zu diesem Zweck sind verschiedene Positionspapiere und Hintergrundinformationen entstanden, die sich überwiegend an das Bundesministerium der Justiz (BMJ), aber auch an die Kommission der Europäischen Union und andere Adressaten richteten. Besonders bedeutsam ist der Schulterschluss mit der Kultusministerkonferenz, der zu einer gemeinsamen Position zur Einführung eines unabdingbaren Zweitveröffentlichungsrechts führte. Diese politische Arbeit war zwar erfolgreich, hat aber bisher noch nicht zum Durchbruch geführt. Sie wird auch künftig einen Schwerpunkt bilden. Auch werden wir die Arbeit der Allianz-Initiative stärker als bisher in die Öffentlichkeit tragen, um Vorwürfen der Untätigkeit der Wissenschaftsorganisationen zu begegnen.

Unsere Anliegen und Desiderate für einen Dritten Korb des Urheberrechtsgesetzes an das BMJ haben wir im Jahr 2010 zusammengestellt (vgl. Anhang). Ich möchte zunächst noch einmal die beiden Punkte verdeutlichen, um die es der Wissenschaft vor allem geht:

- Es müssen zunächst Regelungen gefunden werden, welche die Nachnutzung wissenschaftlicher Literatur für Lehre und Forschung genehmigungsfrei ermöglichen. Alles andere schränkt die moderne digitale Kommunikation, auf die die Wissenschaft angewiesen ist, in unzulässiger Weise ein.

- Dann müssen die Kosten gegenüber den Verlagen in den Blick genommen werden. Kosten sollten nicht beim letztlichen Nutzer, also bei Wissenschaftlerinnen und Wissenschaftlern, Studierenden oder deren Einrichtungen anfallen und dort zu einer Einschränkung der Informationsversorgung führen. Eine Abrechnung gerechtfertigter anfallender Kosten sollte generell über Verwertungsgesellschaften erfolgen - wie dies bereits heute beispielweise mit der Kopierabgabe für Druckerzeugnisse praktiziert wird. Außerdem sollten die Kosten transparent und angemessen sein.

Weder die Genehmigungsfreiheit noch die Kostenfrage sind im geltenden Urheberrechtsgesetz angemessen abgebildet. Eine Revision muss mindestens diese beiden Punkte wie folgt adressieren.

Die Nutzung digitaler Inhalte müsste künftig für alle Zwecke des Unterrichts genehmigungsfrei ermöglicht werden, und nicht wie heute nur für den Unterricht selbst. Dies ist vor allem für Hochschulen von herausragender Bedeutung, weil hier der Anteil des Selbststudiums naturgemäß hoch ist. Auch die in der gültigen Fassung der Urheberrechtsgesetzes benutzten Begrifflichkeiten des „kleinen Teils“ bzw. der „Teile“ eines Werks sind revisionsbedürftig. Hier müsste der Gesetzgeber die Definition dessen, was ein „kleiner Teil“ bzw. ein „Teil“ eines Werkes umfasst, genauer und deutlich großzügiger im Sinne der Wissenschaft verankern. Die bisherige Rechtsprechung geht von maximal 10 Prozent und weniger als 100 Seiten für „kleine Teile“ und von maximal 33 Prozent für „Teile“ eines Werks aus. Dies wird den Nutzungsansprüchen in Lehre und Forschung deshalb nicht gerecht, weil es hier immer um Sinnzusammenhänge geht, die sich nicht durch Seitenzahlen abbilden lassen. Außerdem muss es möglich sein, beispielsweise für quantitative sprachwissenschaftliche Analysen mittels Textmining auch ganze Werke zu nutzen und zu bearbeiten. Daher müssen diese Einschränkungen eigentlich ganz entfallen.

Neben der Kostenfreiheit für die unmittelbaren Nutzer geht es auch und nicht zuletzt um faire und angemessene Erwerbskosten für wissenschaftliche Literatur und um eine Begrenzung des mit der Umsetzung von Nutzungsregularien verbundenen Aufwands.

Die nicht mehr nachvollziehbaren Preissteigerungen der großen Wissenschaftsverlage von teilweise mehr als 500 Prozent in den letzten 10 bis 15 Jahren sind bekannt. Der daraus resultierende Kostendruck auf die wissenschaftlichen Bibliotheken und damit auf die öffentliche Hand kann heute nur unzureichend durch die Bündelung der Nachfragemacht in National- oder Allianzlizenzen gemildert werden. Gesetzgeberisch ist dagegen kein Kraut gewachsen. Ansatzpunkte ergäben sich höchstens kartellrechtlich. Es wäre ggf. einmal lohnend, diesen Weg zu beschreiten. Zumindest sollte im Urheberrechtsgesetz die Möglichkeit zur kostenlosen Bereitstellung von Inhalten auf Repositorien geschaffen werden. Die Wissenschafts- 
organisationen verfolgen auch das Ziel, den für gedruckte Medien geltenden ermäßigten Mehrwertsteuersatz auch auf digitale Medien zu übertragen. Hier gab es in der letzten Zeit ermutigende Aussagen der EU und auch der SPD.

$\mathrm{Zu}$ den völlig dysfunktionalen Bürokratiekosten im Zusammenhang mit der Nutzung digitaler Informationen möchte ich auf zwei Aspekte zur Illustration eingehen:

- Die von der neuen EU-Richtlinie zu den verwaisten Werken geforderte „sorgfältige Suche“ nach Urhebern könnte bei einer engen Auslegung der Sorgfaltspflicht entweder zu erheblichen Mehrkosten im Personalbereich der Bibliotheken oder zu einer schleppenden Umsetzung der Richtlinie selbst führen. Der deutsche Gesetzgeber sollte also bei der Umsetzung der Richtlinie von Beginn an klare Standards definieren, die die Obergrenze des Zumutbaren flach halten. Aus unserer Sicht bietet sich dafür eine einfache Anzeigepflicht für Bibliotheken an.

- Der Versand von Kopien durch die Bibliotheken darf nicht durch eine langwierige Suche nach Verlagsangeboten, die gegebenenfalls zu „angemessenen“ Kosten bereitstehen, verkompliziert und verteuert werden. Hier ist der vorgeschlagene generelle Weg eines Image Scans die richtige Lösung.

Der Schwerpunkt unseres Einsatzes für ein wissenschaftsfreundliches Urheberrecht liegt derzeit auf der Verankerung des bereits erwähnten unabdingbaren Zweitverwertungsrechts für unselbständig erschienene Werke in Paragraph 38 UrhG. Das Zweitverwertungsrecht soll nach einer sechsmonatigen Embargofrist für alle Fächer greifen, und zwar formatgleich mit der Verlagspublikation. Die Notwendigkeit einer rechtlichen Absicherung dieser Praxis ergibt sich daraus, dass Wissenschaftlerinnen und Wissenschaftler aus Angst vor einer rechtlich nicht zulässigen Praxis derzeit auf eine zusätzliche Bereitstellung ihrer Arbeiten in Repositorien oft verzichten. Ein unabdingbares Zweitverwertungsrecht stärkt mithin die Position des Urhebers gegenüber den Verlagen. Dabei geht es wohlgemerkt um ein zusätzliches Recht für Urheber, nicht um einen Zwang, solange es sich um Publikationen aus der grundfinanzierten Tätigkeit von Wissenschaftlerinnen und Wissenschaftlern handelt. Hier gilt die volle Wissenschaftsfreiheit. Wir unterstützen jedoch die Forderung, dass Forschungsförderer wie die DFG, das BMBF und die Europäische Union eine frei zugängliche digitale Kopie von Publikationen, die in drittmittelfinanzierten Projekten entstehen, verpflichtend in ihre Förderbestimmungen aufnehmen.

Außerdem fordert die Allianz ein Recht der Bibliotheken, digitale Kopien im Zusammenhang mit der Langzeit- archivierung, der Bestandserhaltung und Bestandspflege, dem Aufbau digitaler Lehr- und Lernplattformen und virtueller Forschungsumgebungen zu erstellen. Im Falle der Bestandsmaßnahmen sollte dies auf jeden Fall kostenfrei gesichert sein.

Alle diese Regelungen sollten in einer übergreifenden und klar formulierten Wissenschaftsschranke zusammengefasst werden, um den Nutzern einen einfacheren $\mathrm{Zu}$ gang zum UrhG zu ermöglichen. Für eine solche Schranke hat die Allianz den im Anhang abgedruckten Formulierungsvorschlag vorgelegt, der gegenüber der Formulierung des Aktionsbündnisses für Urheberrecht in Bildung und Wissenschaft etwas konservativer und komplizierter daherkommt, aber alle Forderungen an ein modernes Urheberrecht beinhaltet und möglicherweise politisch eher umsetzbar ist. Diese allgemeine Wissenschaftsschranke wäre auf alle Fälle durch die Ermächtigung des Art. 5 Abs. 3 a der Richtlinie 2001/29/EG vollständig gedeckt.

Diese drei Beispiele zeigen die Vielfalt, aber auch die Komplexität der Themen im Umfeld des Urheberrechts, mit denen wir uns intensiv befassen müssen. Wir dürfen nicht weiter hinter die sich rasch entwickelnden technischen Möglichkeiten zurückfallen, wenn wir im globalen Wettbewerb bestehen wollen.

\section{Aktuelle Empfehlungen des Wissenschaftsrates}

Abschließend möchte ich noch kurz über aktuelle Empfehlungen des Wissenschaftsrates zur Weiterentwicklung der wissenschaftlichen Informationsinfrastrukturen in Deutschland bis 2020 berichten. ${ }^{13}$ Zwar sind die Überschneidungen mit dem Thema Urheberrecht nicht besonders groß, dennoch glaube ich, dass die strategische Weiterentwicklung von Informationsinfrastrukturen ein relevantes und drängendes Feld ist, das für Fachleute sicher auch von hohem Interesse ist. Funktionale und qualitativ hochwertige Informationsinfrastrukturen sind unbestritten eine entscheidende Voraussetzung für ein wettbewerbsfähiges Wissenschaftssystem im digitalen Zeitalter. Der Wissenschaftsrat geht in seiner Empfehlung davon aus, dass die strategische Weiterentwicklung der Informationsinfrastrukturen in erster Linie vom Bedarf der Wissenschaft ausgehen sollte, ohne den Bedarf anderer Nutzergruppen,

13 Wissenschaftsrat: Empfehlungen zur Weiterentwicklung der wissenschaftlichen Informationsinfrastrukturen in Deutschland bis 2020, Köln 2012. http://www.wissenschaftsrat.de/download/archiv/ 2359-12.pdf. 
wie z. B. der Industrie, aus dem Blick zu verlieren. Informationsinfrastrukturen sollten daher gemeinsam von Wissenschaftlern und IT-Spezialisten konzipiert und von Dienstleistern geleitet werden. Oberhalb der bestehenden oder neuen Initiativen, wie z. B. der Allianz-Initiative „Digitale Information“, nestor ${ }^{14}$ oder Pangaea ${ }^{15}$, soll ein Rat für Informationsinfrastrukturen als übergeordnetes Koordinierungs- und Beratungsgremium installiert werden, der aus Nutzern und Anbietern bestehen soll. Er soll u. a. die Offenheit des Systems für neue Entwicklungen garantieren, eine Kartierung der Infrastrukturlandschaft vornehmen, eine wettbewerbliche Auswahl geeigneter Initiativen vorbereiten, Forschungsförderer bei der Formulierung neuer Förderinstrumente beraten sowie die Einbindung deutscher Initiativen in internationale Zusammenhänge sichern.

Der Wissenschaftsrat geht davon aus, dass die Gewährleistung einer funktionalen Informationsinfrastruktur eine öffentliche Aufgabe darstellt. Bund und Länder müssen hierfür eine ausreichende institutionelle Grundfinanzierung vorsehen. Eine dynamische Weiterentwicklung entsprechend den Bedarfen des Wissenschaftssystems muss dabei genauso berücksichtigt werden wie ansteigende Betriebskosten und im Laufe der Jahre steigende Reinvestitionskosten. Zwar sind Einsparpotenziale durch eine verstärkt kooperative und arbeitsteilige Servicegewährleistung zu erwarten, dennoch - und dies ist ein klares Signal an die Politik - werden die Ausgaben in diesem Bereich insgesamt in relevantem Umfang gesteigert werden müssen. Bund und Länder sind u.a. aufgefordert, geeignete Finanzierungsmodelle zu entwickeln, die eine Übernahme von projektförmig begonnenen Initiativen in eine Dauerfinanzierung ermöglichen. Eine ausreichende Grundausstattung ist nicht zuletzt auch deshalb erforderlich, um gegebenenfalls auch länderübergreifend Informationsinfrastrukturen und zugehörige Serviceleistungen wieder verstärkt an Hochschulen etablieren zu können. Angesichts der Dringlichkeit der Thematik hat sich der Ausschuss der Gemeinsamen Wissenschaftskonferenz von Bund und Ländern (GWK) Anfang Oktober 2012 darauf verständigt, eine ad-hoc-Arbeitsgruppe einzurichten, die bis Mai 2013 Vorschläge zur Einrichtung eines Rates für Infor-

14 nestor ist ein Kooperationsverbund mit Partnern aus verschiedenen Bereichen, die alle mit dem Thema „Digitale Langzeitarchivierung " zu tun haben. In nestor arbeiten Bibliotheken, Archive, Museen sowie führende Experten gemeinsam zum Thema Langzeitarchivierung und Langzeitverfügbarkeit digitaler Quellen. (vgl. http://www. langzeitarchivierung.de/Subsites/nestor/DE/Home/home_node. html).

15 Pangaea ist ein Informationssystem für Publikationen und Forschungsdaten über Global Change. http://www.pangaea.de. mationsinfrastrukturen gemäß der Empfehlung des Wissenschaftsrats vorlegen soll.

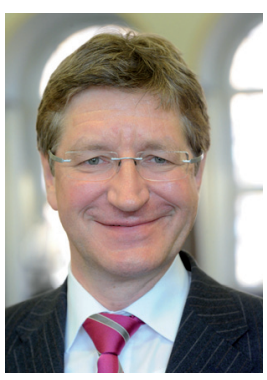

Prof. Dr.-Ing. Wolfgang Marquardt

Geschäftsstelle des Wissenschaftsrates

Brohler Straße 11

D-50968 Köln

Wolfgang.Marquardt@avt.rwth-aachen.de

\section{Anhang}

\section{Wissenschaftsschranke, Formulierungs- vorschlag der Allianz ${ }^{16}$}

\section{$\S 45 b$ Wissenschaftlicher Gebrauch und Bildung}

(1) Für Zwecke des eigenen wissenschaftlichen Gebrauchs und der Bildung ist zulässig

1. zur Veranschaulichung des Unterrichts in Schulen, Hochschulen, in nichtgewerblichen Einrichtungen der Aus- und Weiterbildung sowie in Einrichtungen der Berufsbildung in der für die Unterrichtsteilnehmer erforderlichen Anzahl oder

2. für staatliche Prüfungen und Prüfungen in Schulen, Hochschulen, in nichtgewerblichen Einrichtungen der Aus- und Weiterbildung sowie in der Berufsbildung in der erforderlichen Anzahl

3. für Einrichtungen der wissenschaftlichen Forschung,

- $\quad$ einzelne Vervielfältigungsstücke eines Werkes herzustellen,

- die Vervielfältigung, Verbreitung und öffentliche Wiedergabe eines veröffentlichten Werkes zum Zweck des Zitats, sofern die Nutzung in ihrem Umfang durch den besonderen Zweck gerechtfertigt ist. Zulässig ist dies insbesondere, wenn

1. einzelne Werke nach der Veröffentlichung in ein selbständiges wissenschaftliches Werk zur Erläuterung des Inhalts aufgenommen werden,

2. Stellen eines Werkes nach der Veröffentlichung in einem selbständigen Sprachwerk angeführt werden,

- veröffentlichte Teile eines Werkes, Werke geringen Umfangs sowie einzelne Beiträge aus Zeitungen oder Zeitschriften ausschließlich für einen bestimmt abgegrenzten Kreis von Personen für deren eigene wissenschaftliche Forschung öffentlich zugänglich zu machen, soweit dies zu dem jeweiligen Zweck geboten

16 Allianz der Wissenschaftsorganisationen: Neuregelung des Urheberrechts: Anliegen und Desiderate für einen Dritten Korb, 09.07. 2010. 
und zur Verfolgung nicht kommerzieller Zwecke gerechtfertigt ist.

- auf Einzelbestellung die Vervielfältigung und Übermittlung einzelner in Zeitungen und Zeitschriften erschienener Beiträge sowie erschienene Werke oder Teile davon im Wege des Post- oder Faxversands und in sonstiger elektronischer Form durch öffentliche Bibliotheken, sofern die Nutzung durch den Besteller nach $\S 53$ zulässig ist.

(2) Die Vervielfältigung eines nach Art oder Umfang wesentlichen Teils einer Datenbank ist zulässig zum eigenen wissenschaftli- chen Gebrauch, wenn und soweit die Vervielfältigung zu diesem Zweck geboten ist und der wissenschaftliche Gebrauch nicht zu gewerblichen Zwecken erfolgt.

(3) Vertragliche Regelungen, die urheberrechtliche Schranken für Bildung und Wissenschaft ausschließen, sind unwirksam.

(4) Für Nutzungen zum wissenschaftlichen Gebrauch und für Bildungszwecke ist eine angemessene Vergütung zu zahlen. Der Anspruch kann nur durch eine Verwertungsgesellschaft geltend gemacht werden. 